\title{
R-Sentry: Providing Continuous Sensor Services Against Random Node Failures
}

\author{
Shengchao Yu \\ WINLAB \\ Rutgers University \\ Piscataway, NJ 08854 \\ yusc@winlab.rutgers.edu
}

\begin{abstract}
The success of sensor-driven applications is reliant on whether a steady stream of data can be provided by the underlying system. This need, however, poses great challenges to sensor systems, mainly because the sensor nodes from which these systems are built have extremely short lifetimes. In order to extend the lifetime of the networked system beyond the lifetime of an individual sensor node, a common practice is to deploy a large array of sensor nodes and, at any time, have only a minimal set of nodes active performing duties while others stay in sleep mode to conserve energy. With this rationale, random node failures, either from active nodes or from redundant nodes, can seriously disrupt system operations. To address this need, we propose R-Sentry, which attempts to bound the service loss $d u$ ration due to node failures, by coordinating the schedules among redundant nodes. Our simulation results show that compared to PEAS, a popular node scheduling algorithm, $R$-Sentry can provide a continuous $95 \%$ coverage through bounded recoveries from frequent node failures, while prolonging the lifetime of a sensor network by roughly $30 \%$.
\end{abstract}

\section{Keywords:}

Sensor Networks, Network Coverage, Fault Tolerance, Node Failure, Gang

\section{Introduction}

Sensor networks promise to change the way we interact with the physical world: instead of querying data as a response to events, sensor networks continuously push data to applications so that necessary parsing and analysis can take place before events occur. The very fact that this data may be collected for significant periods of time over vast spatial areas facilitates a broad range of applications. An important issue for the successful deployment of sensor-driven applications is to make sure that the sensor network will be able to deliver as much spatio-temporal information as possible, i.e. sensor networks must guarantee both coverage and con-

\author{
Yanyong Zhang \\ WINLAB \\ Rutgers University \\ Piscataway, NJ 08854 \\ yyzhang@winlab.rutgers.edu
}

nectivity over a significant period of time. Although initial solutions have been proposed to provide coverage and connectivity $[11,4,19,2]$, there is a severe problem- the frequent failing of sensor nodes- that has received little attention. In sensor networks, due to the nature of the sensor node hardware, there exists a fundamental tradeoff between network lifetime and network service quality.

Maintaining graceful operations under faulty conditions has long been a focus of research in other resource-rich systems. Although failures cannot be totally eliminated, a few practical strategies have emerged and been adopted to limit the effects of failures. These strategies usually involve employing backups or redundancy to smoothly transfer the load once a failure occurs. Though effective in resourcerich settings, these strategies can not be applied to sensor networks because of the severe energy constraints, the poor computing capabilities, and the nature of radio circuitry. In particular, many radios employed by today's sensor nodes have the unfortunate failing that, even when idle, they consume nearly as much power as when they are receiving. Further, the power consumption for receiving is almost as taxing as the power needed to transmit. Consequently, turning on backup nodes is not a prudent solution since the backup node might not even outlive the working node.

In order to build a long running system from relatively short-lived sensor nodes, a widely-adopted approach is to include high degree of redundancy in the deployment, and let the nodes work in "shifts". Therefore, at any moment, the working shift (i.e., the active nodes) consists of a minimal number of nodes needed to maintain the system's operations, while the rest of the nodes (i.e., the redundant nodes) have their radios off. The downside of this strategy, however, is that the failure of an active node will lead to a hole in the network, thereby disrupting sensor services. Even in the ideal case where nodes do not die before their batteries drain out, the death of an active node still could disrupt the services since network dynamics make it impossible to precisely predict when power resources will be depleted.

In this paper, we propose $R$-Sentry, a node scheduling algorithm that attempts to balance continuous network ser- 


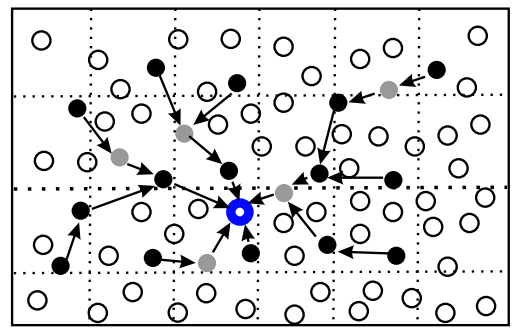

Figure 1. Illustration of a WSN.

vices and extended network lifetime in the presence of frequent node failures. For every active node, R-Sentry groups the nodes whose sensing/networking functionalities overlap with that of the active node into gangs. A gang consists of a set of redundant nodes that can collectively replace the active node upon its failure. R-Sentry ensures that, every so often, a gang will wake up to probe whether the active node is still functioning. If a failure has occurred to the active node, the probing redundant nodes can become active to take over the failed active node to resume network services. Hence, R-Sentry promises to limit the service loss time by coordinating the wake up schedules of the redundant nodes. R-Sentry also seamlessly handles more complicated situations, such as cases where a redundant node serves multiple active nodes simultaneously, or cases where redundant nodes die before the active node fails.

Compared to existing techniques that share the same viewpoint of having redundant nodes sleep for periods of time to conserve energy, such as PEAS [16] or OGDC [19], R-Sentry takes a much closer look at the problem of local network recovery in the face of node failures. It tries to provide quality of service guarantees to sensor applications instead of the best effort approach by using random wake ups. We have conducted a set of simulation experiments to study the performance of R-Sentry. Compared to PEAS, R-Sentry can (1) provide a longer network life, (2) provide better network coverage ( $>95 \%$ ), (3) provide controllable coverage recovery, (4) provide more robustness against random node failures, and (5) provide better scalability with larger node density.

The rest of the paper is organized as follows. We provide an overview of our sensor network model in Section 2, followed by related work. In Section 4, we discuss the RSentry algorithm in detail. We present our simulation effort and the simulation results in Section 5. We conclude in Section 6.

\section{Sensor Network Model}

In this section, we discuss in detail our wireless sensor network (WSN) model.

\subsection{Generic Sensor Network Model}

Coverage: In this work, we adopt the grid-based coverage model $[11,12]$ illustrated in Figure 1. In this popular cover- age model, the square network field in question is imaginarily partitioned into grids, marked by the dotted lines in the figure. The grids should be small enough so that the underlying physical phenomena do not exhibit much variability within a grid. The grid points that fall within a node's sensing area are considered covered by that sensor node. This discretization approach simplifies the measurement of the coverage, making the network coverage percentage equivalent to the percentage of the grid points that are covered. This model can be enforced by having nodes exchange the list of grid points it can cover with their neighbors, which we call GridList. Figure 1 shows such a scenario where there are a subset of sensor nodes, represented by darker solid circles, actively monitoring corresponding grid points, while other nodes are not required for the coverage under the model. With sufficient node density, there is a high probability that, out of uniformly randomly deployed nodes, there exists a set of nodes that could collectively cover all the grid points in the network field.

Connectivity: In addition to sensing the physical world, a WSN is also responsible for delivering the sensed data to the applications. Network connectivity thus requires that there exists a routing path between every sensing node and the sink. In order to achieve this goal, it may be necessary to have more active nodes than just those needed for provide sensor coverage, i.e., we may need extra nodes to have good network connectivity, which are represented by the light solid circles in Figure 1.

To focus on failure recovery schemes, we choose grid sizes small enough to ensure connectivity through coverage: a WSN that satisfies the coverage requirement is automatically connected. This was also observed in many earlier studies [16, 4, 11], which assumed the communication range is at least double of the sensing range. Therefore, in this paper, we can focus on providing sensing coverage.

Turning Off Redundant nodes: Energy is a scarce resource for many sensor nodes may not have external power sources, and have to rely on batteries. Even a battery with a capacity of $3000 \mathrm{~mA}$-hour can only last for 17 months [3]. A sensor node has three main components: sensor(s), processor, and radio. Sensors measure physical phenomena, the processor takes as input the data from the sensors or the network and performs in-network processing, while the radio communicates with the rest of the network. Among the three, the radio is by far the main power consumer [17]. For example, a Mica2 radio has current draw of $12 \mathrm{~mA}$ in transmitting and $8 \mathrm{~mA}$ in receiving. It's worth noticing that a radio being in receiving mode does not necessarily mean the application is receiving any valid packets; it is merely monitoring the medium. The energy consumed by a Mica2 radio in transmitting a 30-byte message is roughly equivalent to the energy consumed by an ATMega128 processor exe- 
cuting 1152 instructions [9]. As a result, if we leave all the sensor nodes on, then no matter how many are deployed, the network cannot function longer than 17 months. In practice, to extend network lifetime it is necessary to have redundant nodes off and turn them back on only when needed.

Disruptions Caused by Node Failures: Although the strategy discussed above is energy-conscious, it does not provide any robustness against random node failures. For instance, suppose that grid point $p$ was monitored by sensor node $s$ while all the other nodes that can also cover $p$ were in sleep mode. Further, suppose that those sleeping nodes will wake up at a later time $t$. If $s$ fails at time $t_{0}$, then during this period with duration $t-t_{0}$, the coverage and/or connectivity of the network will be lost. In particular, node failures will occur more frequently under heavy traffic volumes, which is more likely when the monitored physical phenomena exhibit interesting behavior. Fail to collect or forward data on these occasions can have severe consequences, likely detrimental to the applications.

\subsection{Target Sensor Network Assumptions}

In this study, the WSNs have the following features:

Regularity in Sensing and Communication. The popular disk sensor model $[11,10,16]$ is used to simplify the analysis and simulations. Specifically, a node's sensing area is a circular disk with the node as the center and the sensing range as radius; and the neighbor nodes that fall into the cocentric circle with the transmission range as radius are considered as 1-hop neighbors. Moreover, all the links are symmetric.

Resource-constraints. Sensor nodes are battery-driven and non-rechargeable, except the sink node.

Location-Awareness. Each sensor node is assumed to be stationary and have its own location, which can be obtained through either localization devices, such as GPS, or certain localization algorithms [7, 8]. We further assume the sensor nodes have the geographical location of the network field in which they are deployed. Based on a node's location and sensing radius, it can obtain the list of grid points it covers.

\section{Related Work}

Local networking repair has been studied in the context of sensor networks, and several strategies have been proposed, such as GAF [14], AFECA [13], and ASCENT [1]. GAF identifies those nodes that are equivalent in terms of routing capabilities, and turns them off to conserve energy. In AFECA, a simple adaptive scheme is employed to determine the node sleep interval for those nodes that are turned off. ASCENT shares the same goal as GAF, and it also considers mechanisms for sleeping nodes to come back and join the routing when necessary. To avoid degrading connectiv- ity severely, sleeping nodes need to wake up relatively frequently. In addition, a fixed sleep interval is used for every node in the network. The authors also proved that the upper bound on the lifetime improvement is the ratio of a node's sleep interval to its awake period.

Besides information delivery, data collection is the other critical task of sensor networks. In PEAS [16], an independent probing technique was proposed for redundant nodes to check whether there is an active node in its vicinity. OGDC [19] is a round-based node scheduling algorithm which selects active nodes based on their geo-locations to improve network lifetime. In $[5,15]$, all the nodes that can cover the same spot form a cluster, and at any time, there is only one active node from the cluster while the other members stay in the sleep mode.

Compared to the aforementioned earlier work, R-Sentry takes a closer look at the problem of local network recovery in the face of node failures, and takes a distinctly different approach: (1) R-Sentry tries to provide quality of service guarantees to sensor applications instead of using a best effort approach based on random wake ups; (2) the wake ups of the redundant nodes are carefully scheduled to ensure timely network recovery with less energy consumption, and (3) waking up one redundant node to replace the failed active node is often impossible. R-Sentry extends our exploratory work in [18] by introducing the concept of gangs and conducting more comprehensive evaluations.

\section{The Design of R-Sentry}

While on duty, a WSN is composed of two types of nodes: active nodes, which are performing duties; and redundant nodes, which are sleeping with their radios off. While active nodes is on duty, the redundant nodes should not sleep for an infinitely long period. Rather, they should wake up from time to time and check the conditions of the active nodes. Every node randomly waking up [16, 1], however, is wasteful and does not provide much benefit. For instance, if the subsequent wake ups are far apart from each other, then it is impossible to ensure quick network recovery. To address this void, we propose a coordinated scheduling algorithm among all the redundant nodes. Those redundant nodes that wake up play a role analogous to sentries in real world in the sense that they monitor the health of the active node, and whenever a fault or failure occurs, they jump in to replace the lost node. In the following discussions, we use redundant node and sentry interchangeable. Since redundant nodes rotate to wake up, we call this scheme $R o$ tatory Sentries or R-Sentry.

\subsection{Redundant Sets and Gangs}

Before going into details of R-Sentry, it is beneficial to examine the redundancy among nodes. Every sensor node has a group of neighbor nodes with overlapping communication or sensing capabilities. The sensing redundant set 
$(S R S)$ of a node consists of its neighbors whose sensing areas overlap with the node's sensing area, i.e. nodes that can cover the same grid point(s) belong to each other's SRS.

When an active node fails, it can only be replaced by nodes from its SRS. However, the replacement of a failed active node can not necessarily be accomplished by simply replacing it with a single redundant node, regardless of whether one is trying to repair sensing coverage or network connectivity. This viewpoint is distinctly different from those in earlier studies such as $[16,1]$, which tried to replace the active using one redundant node. In fact, it has been shown in [4] that on average 3-5 nodes are needed to replace a node's sensing area.

Since an active node can only be replaced by certain combinations of redundant nodes, there is a need for the active node to group all the nodes that belong to its $S R S$ into "gangs". Nodes that belong to the same gang can collectively replace the active node. Now let us look at an example to understand the definition of a gang. In this example, the active node $A$ 's $S R S$ is $\{B, C, D, E, F\}$, and their GridLists are shown in Table 1 . We can see that $A$ 's coverage area can be completely replaced by the following combinations: $\{B\},\{C, D\},\{C, E\}$, and $\{D, E, F\}$. As a result, $A$ has four gangs: $\{\{B\},\{C, D\},\{C, E\},\{D, E, F\}\}$, which we call GangList.

One thing we would like to point that is, a superset of a gang set technically is also a gang. For instance, in the example in Table 1 , the set $\{B, C\}$ is also a gang. However, in this paper, we only focus on "minimum gangs", those sets that would no longer be a gang should we remove any member from the set. Grouping nodes from an SRS into gangs is essentially a combinatorial problem. shows the pseudo code for a node populating its GangList with gangs of sizes no larger than gs. In order to limit the computation of this procedure, we only consider gangs of small sizes.

For the purpose of fault tolerance, nodes that belong to a gang need to wake up simultaneously to completely replace the functionalities of an active node.

\subsection{R-Sentry Algorithm}

R-Sentry attempts to bound the duration of service loss. That is, every time an active node fails, R-Sentry seeks to make nodes from a gang available within a time interval of $\Delta$ (an example schedule shown in Table 3(b)), where $\Delta$ is the promised service loss time limit. If an active node has $N$ gangs, then each gang needs to wake up every $N \Delta$.

\begin{tabular}{|l|l||l|l|}
\hline node ID & GridList & node ID & GridList \\
\hline$A$ & $\{1,2,3,4\}$ & $D$ & $\{1,4,5,6,7\}$ \\
\hline$B$ & $\{1,2,3,4,5\}$ & $E$ & $\{3,4,5,6,8\}$ \\
\hline$C$ & $\{1,2,3,5\}$ & $F$ & $\{2,9,10\}$ \\
\hline
\end{tabular}

Table 1. An example of GridList table.

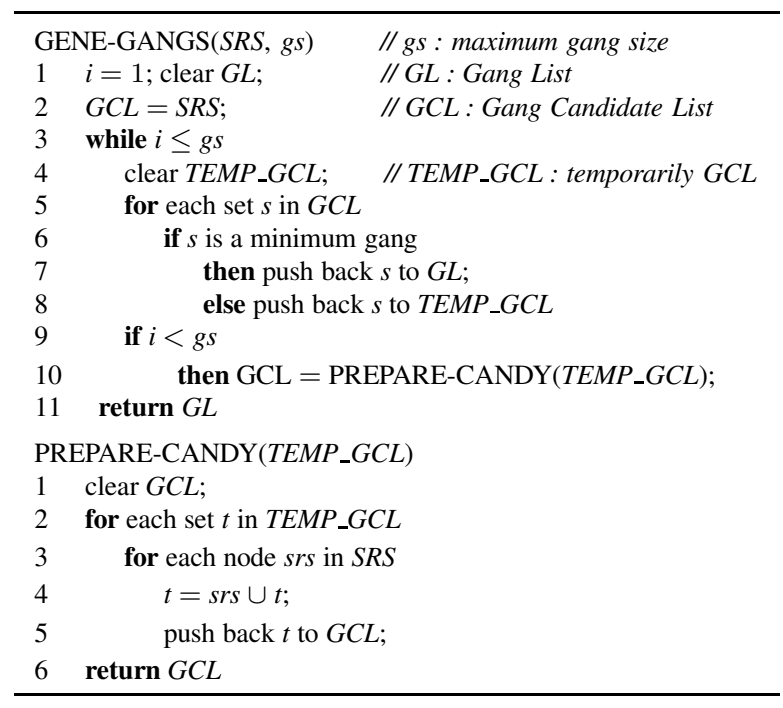

Table 2. Algorithm for generating gang

\begin{tabular}{|l||c|}
\hline Gang & Time To Wake Up (s) \\
\hline \hline$\{B, C\}$ & 1200 \\
\hline$\{D, C, E\}$ & 1500 \\
\hline$\{B, E\}$ & 1800 \\
\hline$\{E, F\}$ & 2100 \\
\hline
\end{tabular}

(a) Gang schedule table



(b) Wake up schedule

\section{Table 3. Illustration of a gang schedule table and the resulting wake up schedule.}

\subsubsection{Basic idea}

The discussions in this section have simplified assumptions such as a redundant node only serves one active node, and all the redundant nodes will not fail before the active nodes do. We made these assumption to keep the explanation easy to understand, and in the following sections, we discuss how to extend R-Sentry to handle more realistic scenarios, where a redundant node may belong to multiple active nodes, and node failures can occur at random times.

We let the active nodes maintain most of the data structures, while having redundant nodes only keep track of their own next wake up time. The most important data structure maintained by an active node is its gang schedule table, which specifies each gang's next wake up time. An illustration of such a data structure is provided in Table 3(a). Based on this table, we can infer the wake up times for each gang. For example, with $\Delta=300 \mathrm{~s}$, if an active node has 3 gangs, the gang that wakes up at time 1200 s also wakes up at times 2100s, 3000s, 3900s, etc. Therefore, a portion of the wake up events caused by Table 3(a) is shown in Table 3(b). 
Next we discuss how active nodes establish their gang schedule tables, and more importantly, how they maintain the schedules.

Initialization: During the bootstrapping phase, a WSN usually runs a host of initialization services, such as neighbor discovery, localization, time synchronization, route discovery, duty-cycle scheduling, etc. Similarly, a WSN that employs R-Sentry needs the following extra initialization services:

- Gang Discovery. This service ensures every sensor node identifies its neighbor nodes, and populates its SRS and GridList. At the beginning, each node in the network sends out a presence announcement message including its ID and location. Such messages are flooded within $h$ hops around the source nodes, where $h$ is a small number, usually 1 or 2 , as the presence announcement only matters to nodes within the vicinity of the source node. After a certain amount of time in the process, every node will receive the announcements from all of its SRS members, based on which a node can calculate the GridList of each node in its $S R S$. After that, a node would be able to form its own GangList in the way illustrated in Table 2.

- Schedule Bootstrapping. The initial set of active nodes are determined by the underlying coverage and initialization protocols. Our approach is similar as the one employed in CCP [11], which can be broken down into 3 steps: 1) after presence announcement exchange phase, every node stays active and starts a random backoff timer, collecting redundancy announcement from its $S R S$ members; 2) upon the timer's expiration, a node checks its $S R S$ members' redundancy status and determines if all grid points in GridList are covered by the non-redundant $S R S$ members. If yes, it considers itself redundant and broadcasts redundancy announcement, otherwise it considers itself non-redundant and doesn't take any actions; 3 ) at the end of bootstrapping phase, the non-redundant nodes calculate their gangs' schedules and flood them within a small number of hops, staying active; while the redundant nodes, upon receiving the schedules, record their own wake up time or the earliest one if it receives multiple schedules.

At the end of the initialization phase, the redundant nodes go to sleep with their sleep timers properly set up, while the active nodes start collecting and forwarding sensed data.

Probing: A sentry (i.e. redundant) node periodically wakes up, as scheduled, to probe the active node. If the active node has failed, sentry nodes will become active to resume network services; otherwise, they go back to sleep.

When a sentry node wakes up, it broadcasts a probing message with its node ID included. Around the same time,



(a) The events that have been observed by $A$.



(b) $t=1200$



(d) $t=1400$

\begin{tabular}{|c|c|}
\hline$\{\mathrm{B}, \mathrm{C}\}$ & 1600 \\
\hline$\{\mathrm{D}, \mathrm{E}\}$ & 1900 \\
\hline$\{\mathrm{E}, \mathrm{F}\}$ & - \\
\hline
\end{tabular}

(f) $t=1550$

\begin{tabular}{|c|c|}
\hline$\{\mathrm{B}, \mathrm{C}\}$ & 2200 \\
\hline$\{\mathrm{D}, \mathrm{E}\}$ & 1900 \\
\hline$\{\mathrm{E}, \mathrm{F}\}$ & 2500 \\
\hline
\end{tabular}

(h) $t=1700$

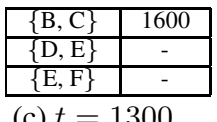

(c) $t=1300$

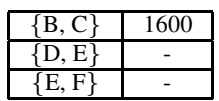

(e) $t=1500$

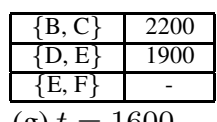

(g) $t=1600$

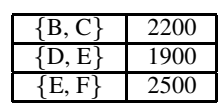

(i) $t=1800$

\section{Figure 2. Illustration of how a new active node establishes its gang schedule table.}

the other sentries of the same gang will also wake up and probe. If the target active node is still alive, it will match the node ID's contained in the probing messages with the gang whose scheduled wake up time is closest to the current time. If the match is successful, the active node updates the gang schedule table by incrementing the wake up time by the round duration $N \Delta$. Finally, the active node sends the sentries a reply message which has two fields: NextWakeTime, and CurrentTime. The CurrentTime field is used to synchronize the clocks between the sentry nodes and the active node.

The above discussion assumes the active node is still alive while sentries probing. If the active node has failed before sentries probe, the sentries will not receive the reply message, then they conclude the active node has failed. In this situation, the sentries should become active to provide uninterrupted services. The design of R-Sentry thus ensures that, whenever one of the active node fails, its functionality will be fully resumed by other nodes roughly within $\Delta$, when the sentries outlive the target active node and corresponding communication time is negligible compared to $\Delta$. Therefore, R-Sentry can limit the service loss period within a tolerable threshold.

\subsubsection{Dynamically establishing schedules for new ac- tive nodes}

After the sentry nodes become active, these new active nodes face several challenges. The main challenge stems from the fact that the communication between the new active node and the redundant nodes that belong to its SRS 
has not been established. On one hand, the redundant nodes are still following the schedules of the previous active node, without realizing that the active node has changed. On the other hand, the new active node does not have a schedule for its gangs, and therefore, it will not be guarded properly. Further complicating the problem is that it is impossible for the new active node to communicate with the redundant nodes when they are asleep for their radios are off. As a result, we can only attempt to establish the schedule gradually as more and more redundant nodes wake up in groups down the stretch.

The algorithm a new active node uses to establish its schedule is rather simple, yet effective. The idea is that, if the redundant node that is probing is not associated with a wake up time in the gang schedule table, the new active node will assign the next available wake up slot to the gang that contains this redundant node. If the node is included in multiple gangs, the smallest gang will be picked.

To better understand this algorithm, let us walk through an example shown in Figure 2. Suppose node $A$ is the new active node, and its GangList is $\{\mathrm{B}, \mathrm{C}\},\{\mathrm{D}, \mathrm{E}\}$, and $\{\mathrm{E}$, $\mathrm{F}\}$. The $\Delta$ is 300 seconds. Figure 2(a) illustrates the wake up events $A$ observes in the establishing phase, and for each event, it shows the corresponding schedule table in the subsequent figures (Figures 2(b-i)). In particular, the establishing phase has the following steps:

1. $A$ becomes active at time 1200 , when $A$ 's schedule is empty (Figure 2(b)).

2. At time $1300, B$ wakes up. $A$ then assigns next available wake up slot, i.e. the current time incremented by $\Delta, 1600$, to gang $\{B, C\}$, and updates the gang schedule table accordingly (Figure 2(c)).

3. At time $1400, C$ wakes up. $A$ finds $C$ is already scheduled, so it does not update the gang schedule table. It just simply sends a reply message to $C$ to instruct $C$ to wake up at 1600 (Figure 2(d)).

4. At time $1500, G$ wakes up. $A$ finds $G$ does not belong to its $S R S$, so it does not update the gang schedule table. $A$ sends a reply message to $G$ with a large sleep interval (Figure 2(e)). If $G$ serves other active nodes, it will receive a much shorter sleep time from them.

5. At time 1550 , node $D$ wakes up. $A$ assigns next available wake up slot, 1900, to gang $\{D, E\}$, and updates the gang schedule table accordingly (Figure 2(f)).

6. At time 1600 , node $B$ and $C$ wake up according to the schedule. Since $A$ 's table is not fully occupied yet, $A$ assigns the next available wake up slot, 2200, to them (Figure 2(g)).

7. At time 1700 , node $F$ wakes up. $A$ assigns the next available wake up slot, 2500, to node $E$ and $F$ (Figure 2(h)).

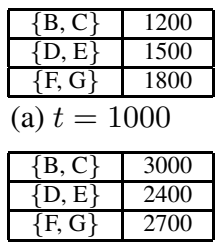

(c) $t=2400, A$ still has not heard from $D$.

\section{Figure 3. An example illustrating how a ac- tive node detects failures among redundant nodes and adapts its schedule accordingly.}

8. At time 1800 , node $E$ wakes up. $A$ does not update the schedule table because $E$ is already scheduled. $A$ sends a reply message to $E$, requesting $E$ to wake up at time 1900 (Figure 2(i)).

9. After that, $A$ becomes a normal active node, and it will handle the subsequent waking sentries by incrementing their wake up times by $3 \Delta=900$.

We note that a new active usually can establish its gang schedule within a reasonable amount of time because according to R-Sentry, every redundant node wakes up periodically, and this period will be the upper bound of the time taken to form the schedule.

\subsection{Scheduling Redundant Nodes That Serve Multiple Active Nodes}

To simplify the discussion, we assumed that a redundant node only serves one active node in the earlier sections. In this section, we look at how R-Sentry handles the cases where a redundant node may serve multiple active nodes.

If a sentry node guards multiple active nodes, the main challenge lies in that when it probes, how it handles schedules from multiple active nodes. In R-Sentry, when a sentry node probes, it only includes its own ID in the probing message. Each of the active nodes that receive the probing messages, will examine the difference between the scheduled wake up time of that redundant node and the current time at the active node. If the difference is below a threshold, the corresponding active node assumes this is a valid wake up, calculates its next wake up time, and sends a reply message back. The reply message contains three fields: the next wake up time $T_{\text {next }}$, the current time $T_{\text {curr }}$, and the active node's ID. Those active nodes that have a different wake up time for the redundant node will simply copy the previously scheduled wake up time to the reply message. After receiving all the reply messages, the redundant node calculates the sleep interval for each of the active nodes, chooses the shortest one as the next sleep interval, and synchronizes its clock appropriately. 


\subsection{Dynamically Adjusting Schedules for Missing Redundant Nodes}

In many sensor network applications, failures occur not only to active nodes, but also to redundant nodes, even when they are in sleep mode. For instance, a catastrophic event, such as lightning can cause sensor node failures, regardless of their state. When a redundant node fails, the active node cannot rely on the gangs that contain the failed node, and should remove these gangs from its schedule.

In R-Sentry, dynamically adapting the active node's schedule is rather straightforward. If the active node does not hear from a redundant node in $k$ consecutive rounds (usually, $k$ is a small number such as 2), it simply removes the gangs that contain the missing node from the gang list. In order to understand the details, let us look at an example illustrated in Figure 3, where the active node $A$ has the following gangs, $\{\mathrm{B}, \mathrm{C}\},\{\mathrm{D}, \mathrm{E}\}$, and $\{\mathrm{F}, \mathrm{G}\}$; and the $\Delta$ is 300 seconds. Node $D$ fails at time 1000 . What happens to $A$ is:

1. At time 1000 , node $D$ fails. $A$ 's gang schedule table is shown in Figure 3(a).

2. At time 1500 (Figure 3(b)), $A$ only receives probes from $E$, not $D$. $A$ decides to wait for one more round (900 seconds in this case) before taking actions.

3. After a round, at time $2400, A$ still has not heard from $D$ (Figure 3(c)). $A$ then concludes that $D$ has failed, and removes the gang $\{D, E\}$ from the schedule (shown in Figure 3(d)). At this time, $A$ will still send a reply message to $E$ that includes a reasonably long sleep time, like $2 \Delta$.

4. $A$ will schedule the remaining two gangs as usual, with the only exception that the round duration now becomes 600 seconds. As a result, gang $\{\mathrm{B}, \mathrm{C}\}$ will wake up at times 3000, 3600, 4200, etc, while gang $\{F, G\}$ will wake up at times $2700,3300,3900$, etc. Therefore, $A$ will still receives probes every 300 seconds.

\section{Performance Evaluation}

In this section, we first define a simplified sensor failure model, then give the simulation model and simulation setups. After that we examine R-Sentry's performance against PEAS, in terms of scalability, energy efficiency, service availability, coverage recoverability and fault tolerance.

\subsection{Sensor Failure Model}

We purposely introduce sensor failures into our simulations to model the fact that random node failures are norms instead of exceptions in sensor network. The catastrophic failure model used in our simulation is a coarse-grained model, in which a percentage of sensor nodes that are alive (but not necessarily active) "die" due to external catastrophic events, like natural disasters. By "die", we mean the node stops functioning completely as an electronic device, even though it's possible in reality that not all components in the node are affected by the external events.

This failure model has two parameters: (i) failure pe$\operatorname{riod} f_{p}$, the mean time between consecutive external catastrophic events (the intervals between two events are random numbers that follow an exponential distribution), and (ii) failure percentage $f_{\%}$, the mean percentage of the live nodes that are affected by a particular event. Upon a catastrophic event, the actual percentage of affected nodes is a random number between 0 and $2 f_{\%}$.

\subsection{Overview of PEAS}

In this study, we compare R-Sentry's performance with PEAS [16], a well-recognized fault-tolerant energyconserving protocol for sensor networks. Before we present the detailed simulation results, we would like to first give a brief description of PEAS. Like R-Sentry, PEAS also assumes that at any moment, only a subset of nodes stay active, while the others go to sleep and wake up periodically to probe the health of the active node(s). PEAS, however, allows the redundant nodes to independently wake up, without coordinating the schedules among them, and as a result, it only provides a coarse granularity of fault-tolerance.

PEAS guarantees there is at least one active node within every redundant node's probing range. An active node controls the wake up frequencies of the redundant nodes using the parameter $\lambda_{d}$, the desired probing rate. Every time an active node receives a probing message, it replies with $\lambda_{d}$ and the actual probing rate $\hat{\lambda}$ that it has observed, based on which the probing node calculates its new probing rate as $\lambda^{\text {new }}=\lambda^{\text {old }} \frac{\lambda_{d}}{\widehat{\lambda}}$. Then the probing node generates its new sleep interval $t_{s}$ following the probability density function $f\left(t_{s}\right)=\lambda^{n e w} e^{-\lambda^{n e w}} t_{s}$. We note that, in PEAS, the parameter $1 / \lambda_{d}$ is similar to $\Delta$ in R-Sentry, both denoting the desired recovery time from the applications, and we use these two notations interchangeably when presenting the results.

\subsection{Simulation Model and Settings}

We have implemented both R-Sentry and PEAS on our own simulator USenSim. USenSim is a discrete event-driven simulator that is intended to model large-scale sensor networks. In order to realize the network scale we envision, i.e. with thousands of nodes, which was impossible with more detailed network simulators such as NS-2, USenSim assumes a constant transmission delay between nodes and serialized transmissions among nodes that compete the channel, similar as the one adopted in [11]. We believe the sim-

\begin{tabular}{|l|l||l|l|}
\hline Routing protocol & Shortest path & Sensing range & $10 \mathrm{~m}$ \\
\hline Communication range & $15 \mathrm{~m}$ & Transmission power & $0.06 \mathrm{w}$ \\
\hline Receiving power & $0.012 \mathrm{w}$ & Idle power & $0.012 \mathrm{w}$ \\
\hline Sleeping power & $0.00003 \mathrm{w}$ & Sensing power & $0.001 \mathrm{w}$ \\
\hline Bandwidth & $20 \mathrm{Kbps}$ & Packet size & $25 \mathrm{Bytes}$ \\
\hline
\end{tabular}

Table 4. Simulation Platform Parameters 


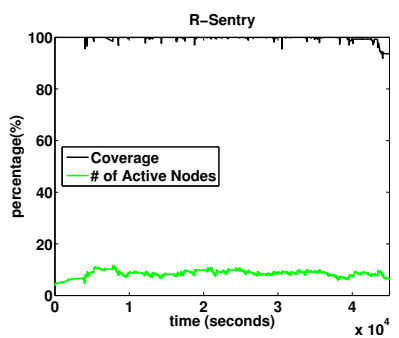

(a)

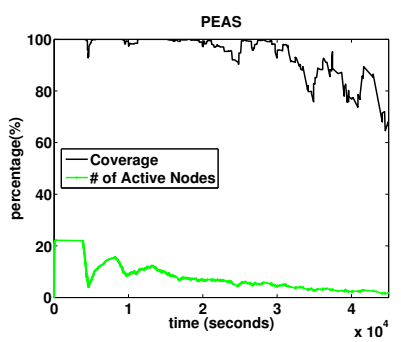

(b)



(c)

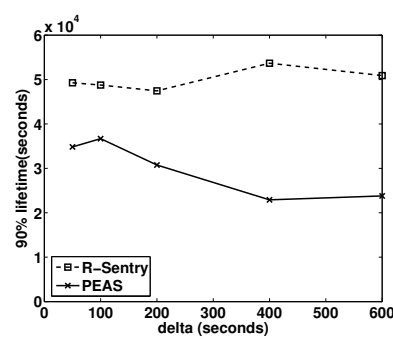

(d)

Figure 4. Network coverage statistics throughout the lifetime of a WSN: (a) R-Sentry, and (b) PEAS. The average coverage loss time and the $90 \%$ network life time with $\Delta$ are shown in (c) and (d).

plified network model does not prevent us proving the validity and effectiveness of our algorithm, given that our work is orthogonal to the works in the underlying layers.

Unless specified, the simulation setup has 600 sensor nodes uniformly randomly deployed in a $50 \times 50 \mathrm{~m}^{2}$ square. Each grid is a $1 \times 1 \mathrm{~m}^{2}$ square. The initial energy of a sensor node is uniformly distributed between 50 and 60 Joules, which allows the node to function around $4000 \sim 5000 \mathrm{sec}-$ onds if the radio is in idle listening mode. Every $50 \mathrm{sec}-$ onds, each active node transmits a packet containing collected data to the sink, which is located at the center of the field and has persistent power supply. The neighbor nodes that are located within the distance of $15 \mathrm{~m}$ constitute $S R S$. Due to the high redundancy, a gang size of 1 is sufficient in the simulations.

For those parameters that are specific to PEAS, we have tried various parameter values, and chosen the following settings because they produced the best results: probing range $R_{p}=3 \mathrm{~m}$, desired probing rate $\lambda_{d}=0.02$, initial probing rate $\lambda_{s}=0.02$, and $k=32$. We would like to emphasize that we chose a rather small probing range for PEAS to guarantee all the grid points are covered since PEAS does not explicitly require every grid point to be covered. The network/energy parameters used in our simulations are summarized in Table 4, and we took these values from PEAS [16].

\subsection{Performance Metrics}

Coverage ratio is the percentage of the grid points that are covered at a time. Both R-Sentry and PEAS attempt to achieve a high coverage ratio throughout their lifetimes.

$\beta$ network lifetime is the duration the network lasts until the coverage ratio drops below $\beta$ and never comes back again. We use $\beta$ lifetime to measure the protocol's capabilities of preserving coverage and recovering coverage loss from node failures.

Coverage loss time is the duration from when a grid point loses coverage to when the coverage recovers. The average coverage loss time reflects how quickly a failed active node can be replaced by the redundant nodes.
Packet delivery ratio is the ratio of number of packets received by the sink to the number of packets sent out by the active nodes in a specific length of time window. The packet delivery ratio indicates the connectivity of the network.

\subsection{Performance Results}

Service Availability: The motivation behind this study is the need to provide uninterrupted services throughout a sensor network's lifetime. Figures 4 (a) and (b) shows how the coverage ratio evolves with time for the two schemes. In this experiment, we have $\Delta=50$ seconds in both schemes. We observe that R-Sentry can offer a $95 \%$ coverage ratio until $2.5 \times 10^{3}$ seconds, while PEAS's coverage ratio drops below $90 \%$ from $2.5 \times 10^{3}$ seconds. This is because in RSentry, once an active node fails, it can be quickly replaced by a gang, while in PEAS, there is not a guarantee that the awake redundant node can fully replace the active node.

We can also confirm our hypothesis by looking at the time series of the percentage of active nodes in both cases. R-Sentry can maintain a steady number of active nodes, which can in turn guarantee a high coverage ratio, but in PEAS, the number of active nodes decreases with time due to oversleeping. We note that the drop of the number of active nodes in PEAS around 5000 second is due to the fact that most of the initial active nodes failed at that time. We did not observe a drop in R-Sentry because it can quickly recover from failures.

Algorithm Controllability: Controllability is a valuable feature for an algorithm, in that the performance of an algorithm with controllability can be easily tuned up to match application requirements. In this set of experiments, we varied $\Delta$, and collected the corresponding average coverage loss time, which is shown in Figure 4(c). We can observe that R-Sentry demonstrates good controllability: for any given $\Delta$ value, the resulting average coverage loss time is always below $\Delta$, and often slightly higher than $\Delta / 2$. PEAS, however, fails to do so - we cannot correlate the delivered coverage loss time and the desirable coverage loss time.

Thanks to the capability of maintaining high coverage ratio and low coverage loss time, R-Sentry can make the sen- 




(a)

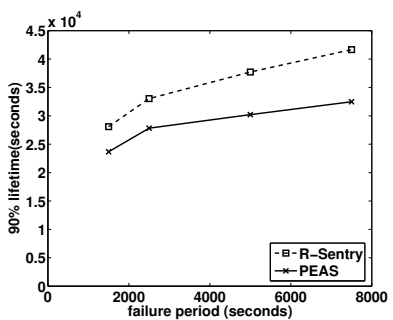

(b)

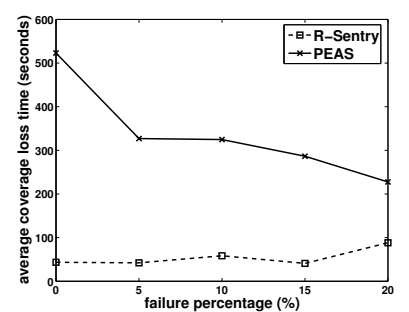

(c)

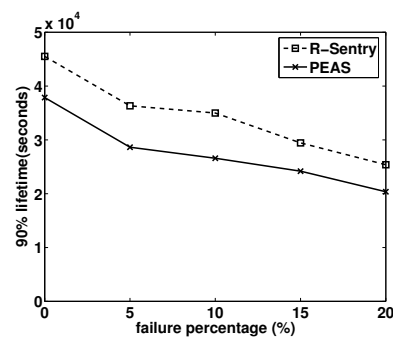

(d)

Figure 5. The impact of $f_{p}$ on average coverage loss time and $90 \%$ network lifetime is shown in (a) and (b) (with $f_{\%}=5 \%$, and $\Delta=50$ seconds). The impact of $f_{\%}$ on average coverage loss time and $\mathbf{9 0 \%}$ network lifetime is shown in (c) and (d) (with $f_{p}=\mathbf{5 0 0 0}$ seconds, and $\Delta=50$ seconds).

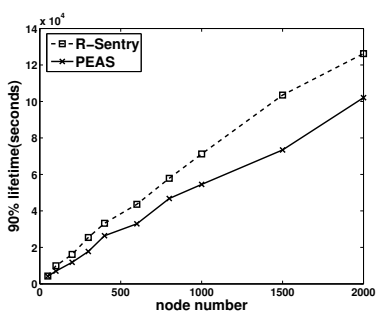

(a)

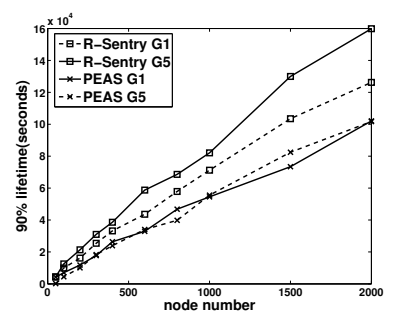

(b)
Figure 6. Scalability and impact of grid size

sor network function for a much longer period, as shown in Figure 4(d). The interesting phenomenon we observe from Figure 4(d) is that the $90 \%$ network lifetime stays almost the same as $\Delta$ goes up. This can be explained by the fact that, though a large $\Delta$ can save more energy by making redundant nodes sleep longer, the likelihood of the overall coverage ratio falling below a certain percentage $(90 \%$ in this case) is also higher due to oversleeping. As a result, these two effects will cancel each other.

Fault Tolerance: It's not unusual that sensor nodes die before running out of energy $[6,16,1]$. In this set of experiments, we evaluate the robustness of the two schemes against node failures under the catastrophic failure model.

In Figures 5(a) and (b), we fixed $f_{\%}$ as $5 \%$, and varied the value of $f_{p}$. Since R-Sentry has sentry nodes guarding active nodes and can dynamically adapt an active's node schedule table to accommodate the failures of its redundant nodes, it is rather robust against random node failures. Figure 5(a) shows that, regardless of the failure rate, R-Sentry is able to replace a failed active node around 50 seconds (which is the value of $\Delta$ ). On the other hand, in PEAS, the average service loss period is much longer. As a result, R-Sentry provides a much better $90 \%$ network lifetime than PEAS across all the failure rates (shown in Figure 5(b)). We also observe the similar trend from the results when we fix $f_{p}$ but vary $f_{\%}$, which are shown in Figures $5(\mathrm{c})$ and $(\mathrm{d})$.

Energy Efficiency and Scalability: Many sensor applications seek to achieve longer network lifetime by deploying more nodes. Therefore, the ability to translate a larger number of sensor nodes into a prolonged network lifetime is critically important to node scheduling algorithms like RSentry and PEAS. In this set of experiments, we varied the number of sensor nodes, and measured the resulting $90 \%$ lifetime. The results, reported in Figure 6(a), show that R-Sentry leads to longer lifetimes than PEAS by roughly $30 \%$. This is because R-Sentry maintains a good coverage ratio for a much longer duration than PEAS through careful scheduling, which leads to a better $90 \%$ lifetime. In fact, we find that the $90 \%$ lifetime in R-Sentry almost scales linearly with the number of nodes, and that the difference between the two schemes increases with the number of nodes.

Connectivity: Our simulations also show that, both algorithms achieve more than $95 \%$ packet delivery ratio during $90 \%$ lifetime, which confirms our claim in Section 2.1. Due to space limitation, we didn't include these plots.

\subsection{Discussion}

Energy Overheard of R-Sentry: The ability to timely wake up appropriate redundant nodes ensures R-Sentry's fault-tolerance. However on the other hand, it also entails additional energy overhead by requiring extra message exchanges between nodes. Assuming no packet loss, when a redundant node wakes up, it will send out a probing message, and will receive $n$ reply messages, where $n$ is smaller than the number of active nodes at the moment. To better understand the overhead, let us next look at one example scenario. We assume 2000 nodes and $1 \times 1 \mathrm{~m}^{2}$ grids, and we further assume that each node on average wakes up 75 times during the $90 \%$ lifetime and receives 16 replies during each wake up (with a total of 1200 reply packets). According to our simulation traces, these numbers are rather conservative. Given the power specifications in Table 4, the amount of energy consumed in transmitting the aforementioned scheduling related packets would be:

$(75+1200) *(25 * 8 / 20000) * 0.06=0.765 \quad$ Joules which is less than $2 \%$ of the initial energy level. Therefore, we take the viewpoint that the energy overhead of R-Sentry is rather low. 
The Impact of Grid Size: The grid is virtual, but its size plays a role in R-Sentry: larger size usually leads to longer lifetime since fewer active nodes are needed to cover all the grid points, which we confirmed through experiments. Specifically, we adopted two grid sizes: $1 \times 1 \mathrm{~m}^{2}$, referred to as $G 1$ and $5 \times 5 m^{2}$, referred to as $G 5$. The results are shown in Figure 6(b). We can see that the $90 \%$ network lifetime of R-Sentry can be further improved by the system adopting a larger grid size. In fact, a grid size of $5 \times 5 \mathrm{~m}^{2}$ extends the lifetime by $30 \%$. However, excessively large grid would compromise connectivity since the network would be disconnected due to low density of active nodes. On the other hand, since PEAS does not rely on the concept of grids, its performance is not influenced by the grid size. We would like to point out that the inconsistency between G1 and G5 in the case of PEAS is caused by artifacts of the simulations.

\section{Concluding Remarks}

Providing continuous, uninterrupted sensor services requires the network to be able to quickly recover from coverage loss due to frequent node failures. That is, if an active node fails, its coverage should be quickly resumed by the redundant nodes, which were sleeping to conserve energy. Earlier node scheduling solutions, such as PEAS [16], adopt completely random schedules among redundant nodes. A random schedule cannot guarantee a redundant node will wake up timely when the active node fails, nor can it guarantee the redundant node that happens to wake up can fully recover the coverage hole.

R-Sentry addresses these issues by grouping redundant nodes into "gangs", which collectively can fully replace an active node, and then by scheduling gangs with fixed intervals. R-Sentry also takes into consideration realistic network situations, such as cases where a redundant node serves multiple active nodes, or cases where redundant nodes may fail before the active node. Through detailed simulations, we show that R-Sentry can provide better resilience against failure while prolonging network life time. As a result, R-Sentry has made a significant step towards building reliable sensor services.

\section{References}

[1] A. Cerpa and D. Estrin. ASCENT: Adaptive SelfConfiguring Sensor Networks Topologies. In Proceedings of IEEE INFOCOM'O2, June 2002.

[2] B. Chen, K. Jamieson, H. Balakrishnan, and R. Morris. Span: An Energy-Efficient Coordination Algorithm for Topology Maintenance in Ad Hoc Wireless Networks. In Proceedings of ACM/IEEE MobiCom 2001, July 2001.

[3] CrossBow Technology. Mote User's Manual. http://www.xbow.com/Support/Support_pdf_files/MPRMIB_Series_User_Manual_7430-0021-05_A.pdf.

[4] Y. Gao, K. Wu, and F. Li. Analysis on the redundancy of wireless sensor networks. In Proceedings of the 2 nd ACM international conference on Wireless sensor networks and applications, pages 108 - 114, September 2003.

[5] T. He, S. Krishnamurthy, J. A. Stankovic, T. Abdelzaher, L. Luo, R. Stoleru, T. Yan, L. Gu, J. Hui, and B. Krogh. An Energy-Efficient Surveillance System Using Wireless Sensor Networks. In Proceedings of the ACM MobiSys 2004, 2004.

[6] W. R. Heinzelman, A. Chandrakasan, and H. Balakrishnan. Energy-Efficient Communication Protocol for Wireless Microsensor Networks. In Proceedings of the 33rd Hawaii International Conference on System Science, 2000.

[7] K. Langendoen and N. Reijers. Distributed localization in wireless sensor networks: a quantitative comparison. Comput. Networks, 43(4):499-518, 2003.

[8] Z. Li, W. Trappe, Y. Zhang, and B. Nath. Robust Statistical Methods for Securing Wireless Localization in Sensor Networks. In Proceedings of the IEEE/ACM IPSN'05, 2005.

[9] S. Mohan, F. Mueller, D. Whalley, and C. Healy. Timing analysis for sensor network nodes of the atmega processor family. In Proceedings of the 11th IEEE Real-Time and Embedded Technology and Applications Symposium, 2005.

[10] D. Tian and N. D. Georganas. A coverage-preserving node scheduling scheme for large wireless sensor networks. In Proceedings of the 1st ACM international workshop on Wireless sensor networks and applications, September 2002.

[11] X. Wang, G. Xing, Y. Zhang, C. Lu, R. Pless, and C. Gill. Integrated coverage and connectivity configuration in wireless sensor networks. In Proceedings of the ACM SenSys'03, pages 28-39, November 2003.

[12] G. Xing, C. Lu, R. Pless, and J. A. O'Sullivan. Co-Grid: an efficient coverage maintenance protocol for distributed sensor networks. In Proceedings of IPSN'04, April 2004.

[13] Y. Xu, J. Heidemann, and D. Estrin. Adaptive energyconserving routing for multihop ad hoc networks. Research Report 527, USC/Information Sciences Institute, 2000.

[14] Y. Xu, J. Heidemann, and D. Estrin. Geography-informed Energy Conservation for Ad Hoc Routing. In Proceedings of the ACM/IEEE MobiCom'01, July 2001.

[15] T. Yan, T. He, and J. A. Stankovic. Differentiated Surveillance Service for Sensor Networks. In Proceedings of the ACM SenSys'03, 2003.

[16] F. Ye, G. Zhong, S. Lu, and L. Zhang. PEAS: A Robust Energy Conserving Protocol for Long-lived Sensor Networks. In Proceedings of ICDCS'03, May 2003.

[17] W. Ye, J. Heidemann, and D. Estrin. An Energy-Efficient MAC Protocol for Wireless Sensor Networks. In Proceedings of IEEE INFOCOM'02, June 2002.

[18] S. Yu, A. Yang, and Y. Zhang. DADA: A 2-Dimensional Adaptive Node Schedule to Provide Smooth Sensor Network Services against Random Failures. In Proceedings of the Workshop on Information Fusion and Dissemination in Wireless Sensor Networks, 2005.

[19] Honghai Zhang and Jennifer C. Hou. Maintaining sensing coverage and connectivity in large sensor networks. Wireless Ad Hoc and Sensor Networks: An International Journal, 1(12), January 2005. 